\title{
CORRECTION
}

\section{Correction to: Improving lifetime of wireless sensor networks based on nodes' distribution using Gaussian mixture model in multi-mobile sink approach}

\author{
Houriya Hojjatinia $^{1}$ - Mohsen Jahanshahi ${ }^{2}$. Saeedreza Shehnepoor ${ }^{3}$
}

Published online: 3 April 2021

๑) Springer Science+Business Media, LLC, part of Springer Nature 2021

\section{Correction to:}

Telecommunication Systems

https://doi.org/10.1007/s11235-021-00753-6

The original version of this article unfortunately contained a mistake. The first author and second author affiliation details are corrected.

Publisher's Note Springer Nature remains neutral with regard to jurisdictional claims in published maps and institutional affiliations.

The original article can be found online at https://doi.org/10.1007/ s11235-021-00753-6.

\section{Mohsen Jahanshahi}

mjahanshahi@iauctb.ac.ir

Houriya Hojjatinia

houriya.hojjatinia@srbiau.ac.ir

Saeedreza Shehnepoor

saeedreza.shehnepoor@research.uwa.edu.au

1 Department of Computer Engineering, Science and Research Branch, Islamic Azad University, Tehran, Iran

2 Department of Computer Engineering, Central Tehran Branch, Islamic Azad University, Tehran, Iran

3 Department of Electronic, Electrical and Computer Engineering, University of Western Australia, Perth, Australia 